\title{
Managing Mycoplasma genitalium - are we doing enough?
}

Helen Callaby ${ }^{1}$, Nicolas Pinto-Sander ${ }^{2}$, Suneeta Soni ${ }^{1,2}$ \& Daniel Richardson ${ }^{1,2}$

1. Brighton and Sussex Medical School, 2. Brighton and Sussex University Hospitals NHS Trust

\section{Introduction}

Mycoplasma genitalium (MG) causes urethritis in males and cervicitis and PID in females ${ }^{1}$. The prevalence is not well understood and frequent use of single dose macrolide antibiotics is driving antimicrobial resistance ${ }^{2}$.

\section{Aims}

1. To identify if we are doing enough to detect MG

2. To understand how our treatment policies may vary

Of the 461 tests:

Of the 30 positive results:

500

400

300

200

100
0

$$
100
$$

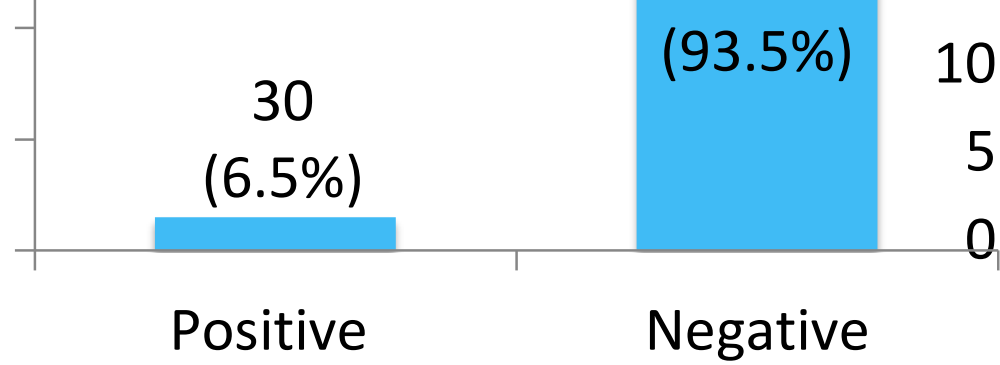

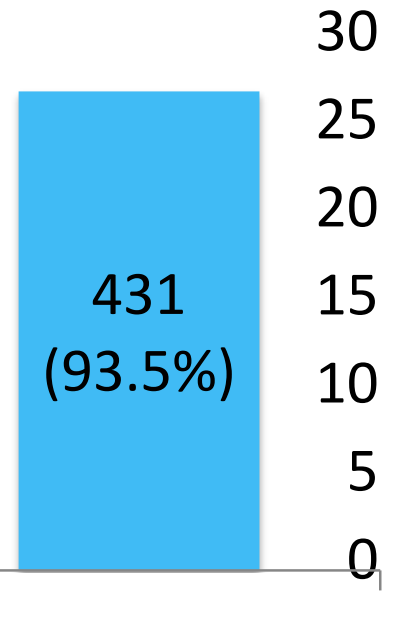

Negative

5

20

\section{0}

$10-4$

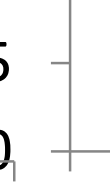

$(13.3 \%)$

Female

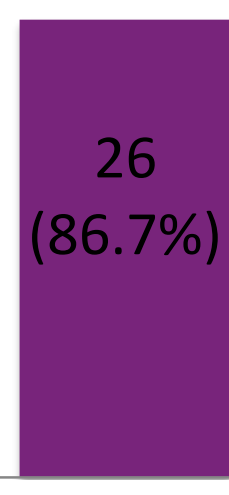

Male

Figure 1: Number of tests conducted and positive results according to sex.

\section{Method}

From November 2011 - May

2015 men with persistent

urethritis or persistent proctitis

and women with persistent PID

were tested for MG using the

Fast-track Diagnostics ${ }^{\mathrm{TM}}$

urethritis PCR. Persistent

symptoms were defined as

recurrent symptoms not

responsive to standard

treatment regimes.

Results: 461 test were done which yielded 30 positive results [Figure 1]. The median age was 30.5 years (range 16-53). All females identified as heterosexual, and of the males; 10/26(38\%) were MSM. 6/30(20\%) patients were known HIV-positive, all of whom were male and 5/6(83\%) were MSM. Test sites according to sex are seen below [Figure 2].

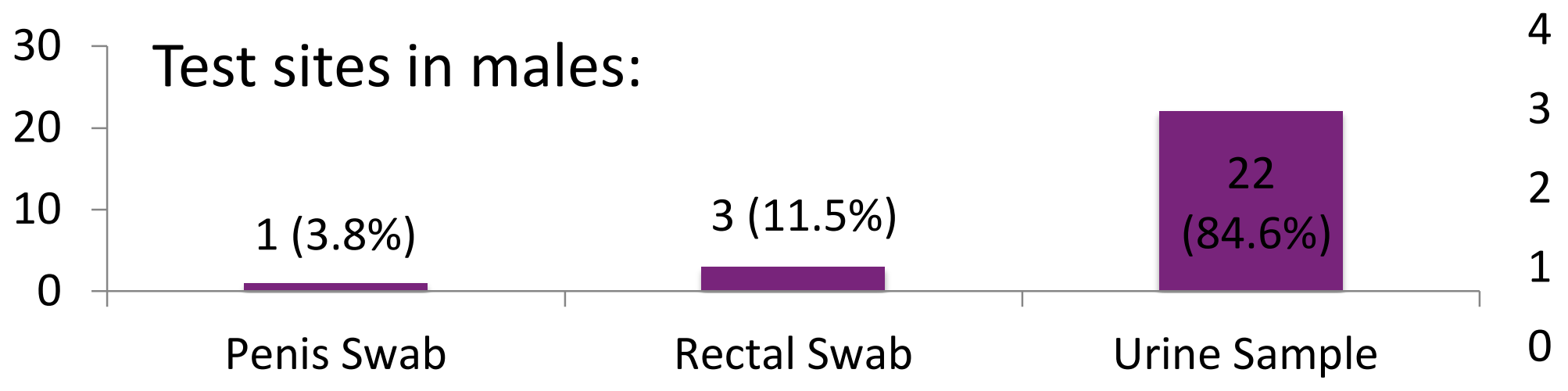

Figure 2: Test sites according to sex.

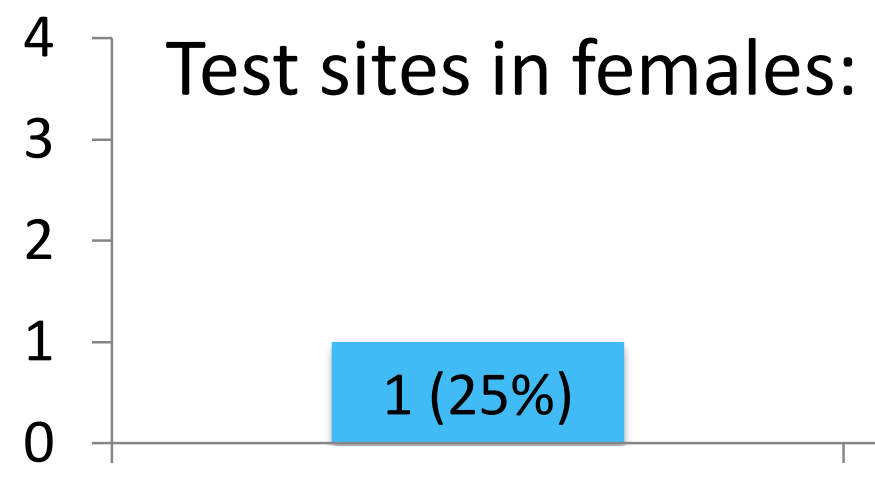

Cervical Swabs
$3(75 \%)$

Vaginal Swabs

$9 / 30(30 \%)$ of all patients were treated with single dose azithromycin $1 g ; 5 / 30(16.7 \%)$ received azithromycin stat followed by a further 4 day course.

Tests of cure were done in $13 / 30(43.3 \%)$ of patients $-4 / 13(30.7 \%)$ patients remained positive and all received Moxifloxacin, which was curative.

\section{Conclusion}

- A number of symptomatic patients had confirmed MG. Nearly one third of patients with MG were inappropriately treated with single dose azithromycin.

- Given the threat of increasing macrolide resistance in this organism local protocols for urethritis and PID should now include routine testing for MG so that appropriate treatment can be prescribed. 